\title{
Cancer Chemotherapy
}

\author{
Philip O. Anderson
}

C LINICIANS INVOLVED WITH BREASTFEEDING have probably heard the generalization that most drugs are compatible with breastfeeding except for cancer chemotherapy and radioactive agents. In fact, as with most broad generalizations, this statement is not entirely correct. In this issue, we examine cancer chemotherapy agents more closely. Radiopharmaceuticals will be discussed in next month's column.

With cancer chemotherapy, the generalization might have been true in the past when cancers were treated with highly cytotoxic agents. Two reports from the 1970's involved mothers receiving cyclophosphamide who breastfed their infants despite warnings to the contrary. One mother was also receiving vincristine. Both of their infants developed neutropenia and one also developed thrombocytopenia and anemia. ${ }^{1,2}$ Usually one would not generalize from two old case reports, but these along with "common sense" have apparently led to the warning against breastfeeding with "cancer chemotherapy." One result of this has been that most drugs used to treat cancer have almost no published information on their use in nursing mothers or even measured concentration in breastmilk that can be used to assess their safety. Only 12 traditional chemotherapy agents (excluding biologicals, tyrosine kinase inhibitors, etc.) have had levels measured in breastmilk, usually in only one or two mothers. ${ }^{3}$

In addition to the treatment of cancer, some chemotherapy agents are used in low doses as immunosuppressants to treat inflammatory diseases such as rheumatoid arthritis and Crohn's disease. Azathioprine and its active metabolite mercaptopurine were originally proscribed during breastfeeding, but slowly case reports, case series, and, eventually, more formal studies found that breastfed infants do just fine when their mothers are taking these drugs in low doses. Now virtually every official guideline for treating these diseases states that breastfeeding is acceptable during therapy with azathioprine or mercaptopurine. This certainly does not extend to high-dose mercaptopurine, now rarely used for treating cancer, but only for low immunosuppressant doses.

An analogous situation exists for methotrexate. Single low doses of methotrexate are used in ectopic pregnancy and low repeated doses are used to treat rheumatoid arthritis and inflammatory bowel disease. A small amount of evidence has been published on the excretion of methotrexate into breastmilk. The first article to report a case was on a patient who was 1 month postpartum given $22.5 \mathrm{mg}$ of methotrexate daily by mouth for choriocarcinoma. Milk was collected at various times of the first 12 days of therapy. The authors estimated that a cumulative amount of $0.32 \mu \mathrm{g}$ would be excreted in milk during the first 12 hours after this dose. The authors concluded that the amount of methotrexate in breastmilk did not appear to constitute a contraindication to breastfeeding, at least in the setting where bottle feeding would be potentially harmful to the infant. ${ }^{4}$ Interestingly, numerous review articles and guidelines cite this article as evidence that breastfeeding is contraindicated during methotrexate therapy, even when given only once or twice weekly in doses similar to those used daily in the case report. Two other reports have measured methotrexate in breastmilk after single doses of 25 and $65 \mathrm{mg}$. Milk levels were at or lower than the limit of detection of $22.7 \mu \mathrm{g} / \mathrm{L}$ in both cases. One mother continued to breastfeed for an additional 9 months while receiving subcutaneous methotrexate $25 \mathrm{mg}$ weekly, with no adverse effects noted in her infant. ${ }^{5}$ Authors of both of these articles felt that breastfeeding was acceptable when the drug was used in low intermittent doses. Clearly, more data are needed before low-dose methotrexate can reach the breastfeeding safety status of low-dose azathioprine.

The most thoughtful and nuanced review of breastfeeding by mothers being treated for cancer appeared in 2013 in Cancer Treatment Reviews. ${ }^{6}$ The authors carefully distinguished between the various classes of chemotherapy agents. They concluded that limited data suggest that antimetabolites (e.g., fluorouracil and low-dose methotrexate) appear to be relatively safe, whereas high-dose methotrexate, anthracyclines (e.g., doxorubicin and mitoxantrone), and alkylating agents (e.g., cyclophosphamide and platinum compounds) should be avoided during breastfeeding or breastfeeding should be withheld during treatment with these agents. The authors also provide some guidance on the time that breastfeeding should be withheld after etoposide: 72 hours after a dose. The authors were not too concerned about monoclonal antibodies. Monoclonal antibodies were discussed in this column in more detail in the April issue of Breastfeeding Medicine. Other targeted therapies such as the tyrosine kinase inhibitors (e.g., imatinib) were also discussed. The authors felt that the low levels of imatinib reported in breastmilk appear to cause no risk to breastfed infants, but that other agents in the class have no data during breastfeeding. With imatinib, withholding breastfeeding for 9 hours after a dose should reduce infant exposure by $25-50 \%$ based on data from one mother. ${ }^{7}$ Taking the dose after the infant's last feeding of the day could be a good strategy if the infant is sleeping through the night. Recently, a woman was reported to have

Skaggs School of Pharmacy and Pharmaceutical Sciences, University of California, San Diego, La Jolla, California. 
breastfed three infants while taking a different tyrosine kinase inhibitor with each pregnancy. None of her breastfed infants appeared to have any adverse drug effects. ${ }^{8}$

Since cancer chemotherapy is often given in cycles, highly motivated nursing mothers sometimes ask whether they can breastfeed in between cycles. As already suggested, this can be a strategy to employ. In the absence of specific patient data, the time to withhold breastfeeding is best determined by using the serum half-life of the drug as a proxy for the halflife of the drug in breastmilk, although they are not always the same. Typically, a period of five half-lives would be considered a safe waiting period. If combination chemotherapy is being given, the drug with the longest half-life would usually determine the waiting period. It is important to consider any active metabolites, also, and use the longest of their halflives. For drugs that are likely not too toxic, a shorter period could be considered. After three half-lives, $87.5 \%$ of the drug is eliminated by the mother, after four half-lives, $94 \%$, and after five half-lives $97 \%$. So, after three half-lives, diminishing returns are gained from longer waiting periods. During the waiting period, milk can be pumped and discarded to maintain the milk supply. Nevertheless, some of the more toxic drugs have long half-lives, making breastfeeding between chemotherapy cycles unfeasible.

A new consideration with regard to cancer chemotherapy is its effects on the microbiome of the breastmilk. Many of the older chemotherapeutic agents have antimicrobial properties that can disrupt the breastmilk flora. In the first case report, a woman was receiving doxorubicin, bleomycin, vinblastine, and dacarbazine for treatment of Hodgkin's lymphoma and was told that she could resume breastfeeding 12 days after each round of chemotherapy. ${ }^{9}$ She was followed for 4 months. Milk samples taken over 4 months indicated a depletion of Bifidobacterium, Eubacterium, Staphylococcus, and Cloacibacteriumin species, whereas an increase of Acinetobacter, Xanthomonadaceae, and Stenotrophomonas species. The beneficial metabolites docosahexaenoic acid and inositol were also decreased. It is not clear what these changes in microbiome mean clinically or how generalizable this case is, because both doxorubicin and bleomycin have antibiotic properties. Whether nonantibiotic chemotherapy alone would cause the same antimicrobial disruption is not known.

Two centers are collecting milk samples and case information from nursing mothers taking medications under IRBapproved protocols. Clinicians who have contact with nursing mothers taking chemotherapy who are willing to provide milk samples are urged to contact one of these centers. Mothers taking intermittent, low-dose methotrexate are of particular interest, but more information on all chemotherapy agents is needed.

All drugs: Kerri Bertrand, MPH, Research Coordinator, MotherToBaby CA, University of California San Diego,
Department of Pediatrics, Division of Dysmorphology. E-mail: kdutcher@ucsd.edu

Methotrexate: Shinya Ito, MD, Division Head, Clinical Pharmacology \& Toxicology, The Hospital for Sick Children, Department of Paediatrics, E-mail: shinya.ito@ sickkids.ca

Currently, LactMed contains records on all cancer chemotherapy with published information and closely related drugs. Drugs with no information at all are not currently listed, but should be considered to be potentially hazardous to breastfed infants. LactMed contains records on all marketed monoclonal antibodies.

\section{References}

1. Durodola JI. Administration of cyclophosphamide during late pregnancy and early lactation: A case report. J Nat Med Assoc 1979;71:165-166.

2. Amato D, Niblett JS. Neutropenia from cyclophosphamide in breast milk. Med J Aust 1977;1:383-384.

3. Anonymous. LactMed. National Library of Medicine. National Institutes of Health. http://toxnet.nlm.nih.gov/cgi-bin/ sis/htmlgen?LACTMED, accessed March 24, 2016.

4. Johns DG, Rutherford LD, Leighton PC, et al. Secretion of methotrexate in human milk. Am J Obstet Gynecol 1972; 112:978-980.

5. Thorne JC, Nadarajah T, Moretti M, et al. Methotrexate use in a breastfeeding patient with rheumatoid arthritis. J Rheumatol 2014;41:2332.

6. Pistilli B, Bellettini G, Giovannetti E, et al. Chemotherapy, targeted agents, antiemetics and growth-factors in human milk: How should we counsel cancer patients about breastfeeding? Cancer Treat Rev 2013;39:207-211.

7. Gambacorti-Passerini CB, Tornaghi L, Marangon E, et al. Imatinib concentrations in human milk. Blood 2007;109: 1790.

8. Alizadeh H, Jaafar H, Kajtar B. Outcome of 3 pregnancies in a patient with chronic myeloid leukemia who received 3 types of tyrosine kinase inhibitors each in different pregnancy: Follow-up of the case with a review of published reports. Ann Saudi Med 2015;35:468-471.

9. Urbaniak C, McMillan A, Angelini M, et al. Effect of chemotherapy on the microbiota and metabolome of human milk, a case report. Microbiome 2014;2:24.

Address correspondence to: Philip O. Anderson, PharmD

Skaggs School of Pharmacy and Pharmaceutical Sciences University of California, San Diego 9500 Gilman Drive La Jolla, CA 92093-0657

E-mail: phanderson@ucsd.edu 